\title{
Demographic profile and predictors of unmet need for family planning among Jordanian women
}

\author{
Salah Mawajdeh
}

\begin{abstract}
Background Despite a reduction in fertility rates and a rise in the prevalence of contraceptive use in Jordan, a gap still exists between women's knowledge of family planning methods and actual usage of these methods. The study aimed to measure the prevalence of unmet need, describe the main socioeconomic characteristics, and outline the predictors of unmet need.
\end{abstract}

Methods A nationally representative sample of 2406 women using Ministry of Health facilities was obtained by applying a two-stage cluster sampling technique. Six strata were identified based on three geographical regions and two types of health centre.

Results The study results demonstrate the overall prevalence of unmet need to be $16.3 \%$, comprising $6.8 \%$ of women who wanted to limit births and $9.5 \%$ who wanted to space births. A negative significant correlation was found between unmet need and the women's educational level. There was a higher level of unmet need in the south compared to other regions. Women's age categories showed high unmet need in the young and old categories compared to the middle age category of 25-35 years of age. A similar pattern was also seen when the number of living children was considered.

Conclusions The study results indicate that lowering the prevalence of unmet need in Jordan is dependent on reducing the knowledge gap, since unmet need is related to women's level of education. Improving access to services at the regional level is also a key factor in reducing disparities between geographical regions.

Keywords family planning, primary health care, reproductive health services, unmet need

J Fam Plann Reprod Health Care 2007; 33(1): 53-56

(Accepted 4 August 2006)

\section{Introduction}

Jordan is a small Middle Eastern country with a population of 5.04 million individuals (cf. 1 million in 1960)..$^{1}$ Almost $80 \%$ of the population live in urban settlements. More than $95 \%$ of adult males are literate compared to $85 \%$ of females. ${ }^{1}$ The annual population growth rate in 1950 was 2.5 compared to 3.1 in $1960,3.4$ for the period 1986-1990 and 2.8 in $2000 .^{2-5}$ The total fertility per woman aged 15-49 years decreased from 6.6 children in 1983 to 5.6 in 1990 and 4.4 in $1997 .^{2-4}$

In Jordan, results from the 2002 Jordan Population and Family Health Survey (JPFHS) indicated that 56\% of married women were using a contraceptive method, and $41 \%$ of current users relied on modern methods. ${ }^{6}$

The 2002 JPFHS indicated that $11.1 \%$ of currently married women were in need of family planning, a $50 \%$ decline from the 1990 JPFHS figure of $22.4 \%$. The unmet need for spacing births and limiting births was $5.6 \%$ and $5.5 \%$, respectively. According to the 1997 JPFHS, of the $53 \%$ of women using contraception, $18 \%$ reported using it to delay the birth of their next child, while $34 \%$ wanted to stop having children.

The present study was designed to assess prevalence, demographic characteristics and predictor variables of unmet need. Documenting the unmet need for family planning among users of Ministry of Health (MOH) services and factors influencing it can assist in developing programmes that target high-priority subgroups and in creating markets for available family planning methods.

\footnotetext{
Faculty of Medicine, Jordan University of Science and Technology, Amman, Jordan

Salah Mawajdeh, MD, DrPH, Associate Professor of Health Policy and Management
}

Correspondence to: Dr Salah Mawajdeh, Faculty of Medicine, Jordan University of Science and Technology, PO Box 811951, Amman 11181, Jordan. E-mail: fhg@index.com.jo

\section{Key message points}

- Despite a reduction in fertility rates and a rise in the prevalence of contraceptive use in Jordan, a gap still exists between women's knowledge of family planning methods and actual usage of these methods.

- Lowering the prevalence of unmet need in Jordan is dependent on reducing the knowledge gap, since unmet need is related to women's level of education.

- Improving access to services at the regional level is a key factor in reducing disparities between geographical regions.

\section{Methods}

The study followed a cross-sectional design using a structured, closed questionnaire. For the purposes of the study, the published literature standard definition of unmet need was used.?

A two-stage stratified cluster sampling design was applied. The country was divided into six strata based on the administrative distribution of three regions (northern, central and southern) and the two types of health facilities (comprehensive health centres and primary health centres). Thus each stratum included a single type of health centre in a specific region.

Any married woman of reproductive age visiting a health centre for any reason (whether seeking medical services, reproductive services or child health care services) qualified for selection. All women who registered on a particular day were recorded on a pre-prepared sheet and the total number of registered women was used as a denominator for weighting purposes in the data analysis. Since this was a facility-based study, women who did not use $\mathrm{MOH}$ services had no chance of being selected, and thus the study findings are not generalisable beyond $\mathrm{MOH}$ clients.

\section{Ethical approval}

The Ministry of Health in Jordan does not have a research committee as such, however the study protocol, 


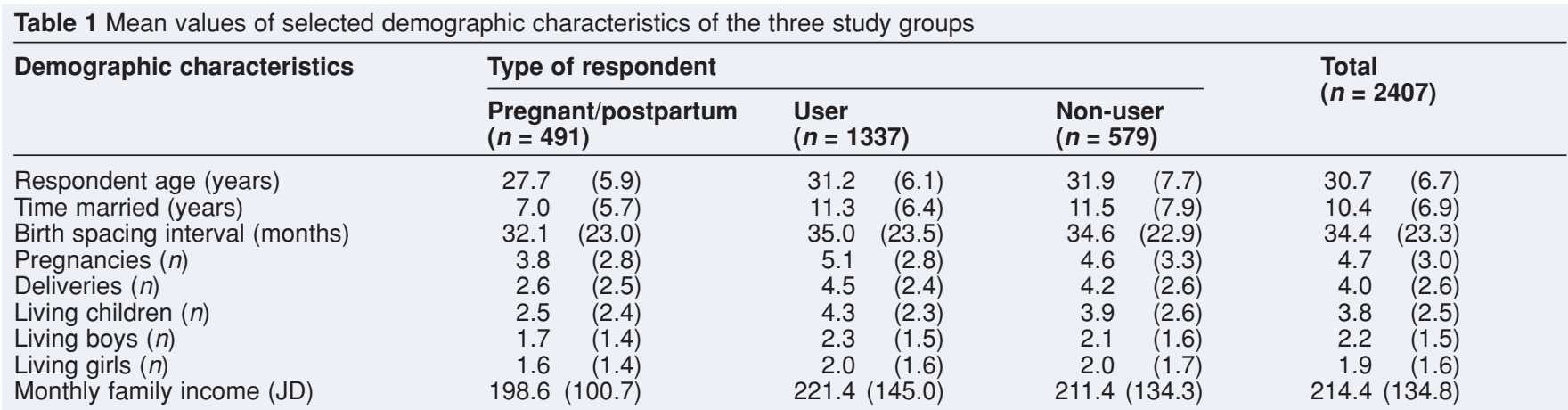

Standard deviation given in parentheses. JD, Jordanian Dinars.

research instruments and consent form were reviewed by a special committee of key decision-makers in the Ministry of Health. Women were given the opportunity to participate in the study after reading and signing the consent form.

\section{Results}

The study results are based on face-to-face interviews with 2406 women subdivided into the following three categories: pregnant/postpartum $(20.4 \%)$, users of family planning methods $(55.6 \%)$ and non-users of family planning methods $(24.1 \%)$.

Women in the study had been married for approximately 10 years and had a mean age of 30.7 years. On average, women in the study reported having been pregnant 4.7 times, with an average of 4.0 deliveries. The average monthly family income of the interviewed women was Jordanian Dinars (JD) 214.4 (Table 1).

The study results showed that the overall prevalence of unmet need was $16.3 \%$, comprising $9.5 \%$ unmet need for birth spacing and $6.8 \%$ for birth limiting. Analysis of the prevalence of standard unmet need by type of respondent showed that standard unmet need for pregnant/postpartum women was $15.9 \%$ (95\% CI 12.3-17.4). The corresponding standard unmet need for non-users was $54.4 \%$ (95\% CI 51.3-58.2).

When standard unmet need prevalence was analysed by selected demographic characteristics the study results showed that older women (aged over 35 years) had the highest standard unmet need prevalence $(21.2 \%)$ compared to $17.7 \%$ for women below the age of 25 years and $13.1 \%$ for the group of women aged between 25 and 35 years $(p<0.001)$. When standard unmet need was examined in relation to the number of living children, the study results showed that the highest prevalence of unmet need (20.0\%) was observed in women who had five or more children $(p<0.001)$. No apparent trend was noted when income categories were compared $(p<0.056)$.

Table 2 also shows that illiterate women had the highest unmet need prevalence $(30.2 \%)$ when compared to women who had some form of schooling $(16.1 \%)$ or women with higher education $(13.8 \%)(p<0.0001)$. Women with health insurance had higher unmet need than women with no health insurance $(p<0.001)$. In addition, women living in the southern region had the highest standard unmet need $(26.7 \%)$ compared to women living in the northern $(18.7 \%)$ or the central region $(11.9 \%)(p<0.0001)$.

Analysis of the types of unmet need (whether for the

Table 2 Distribution of unmet need by explanatory variables

\begin{tabular}{|c|c|c|c|c|c|c|c|c|}
\hline \multirow[t]{2}{*}{ Explanatory variables } & \multirow[t]{2}{*}{$n$} & \multicolumn{2}{|c|}{ Unmet need } & \multicolumn{2}{|c|}{ Spacing } & \multicolumn{2}{|c|}{ Limiting } & \multirow[t]{2}{*}{$p$} \\
\hline & & $n$ & $\%$ & $n$ & $\%$ & $n$ & $\%$ & \\
\hline Total $(n)$ & 2407 & $393^{a}$ & 16.3 & 227 & 9.5 & 165 & 6.8 & \\
\hline \multicolumn{9}{|l|}{ Respondent age (years) } \\
\hline $\begin{array}{l}<25 \\
25-35\end{array}$ & 477 & 84 & 17.7 & 80 & 16.9 & 4 & 0.8 & \\
\hline $\begin{array}{l}25-35 \\
>35\end{array}$ & 1238 & 162 & 13.1 & 120 & 9.7 & 42 & 3.4 & 0.001 \\
\hline$>35$ & 684 & 145 & 21.2 & 26 & 3.8 & 119 & 17.4 & \\
\hline \multicolumn{9}{|l|}{ Living children $(n)$} \\
\hline$<3$ & 778 & 123 & 15.9 & 117 & 15.1 & 6 & 0.8 & \\
\hline $3-4$ & 839 & 111 & 13.3 & 72 & 8.6 & 39 & 4.7 & 0.001 \\
\hline$\geq 5$ & 791 & 158 & 20.0 & 39 & 4.9 & 120 & 15.1 & \\
\hline \multicolumn{9}{|l|}{ Income quantiles (JD) } \\
\hline$\geq 130$ & 444 & 81 & 18.3 & 49 & 10.9 & 33 & 7.4 & \\
\hline $131-159$ & 476 & 83 & 17.4 & 40 & 8.5 & 42 & 8.9 & \\
\hline $160-200$ & 643 & 116 & 18.1 & 77 & 12.0 & 39 & 6.1 & 0.056 \\
\hline $201-260$ & 279 & 30 & 10.9 & 21 & 7.4 & 10 & 3.5 & \\
\hline$>260$ & 471 & 74 & 15.7 & 35 & 7.5 & 38 & 8.2 & \\
\hline \multicolumn{9}{|l|}{ Education (n) } \\
\hline Illiterate & 123 & 37 & 30.2 & 10 & 8.3 & 27 & 21.9 & \\
\hline School & 1753 & 282 & 16.1 & 164 & 9.4 & 118 & 6.7 & 0.0001 \\
\hline $\begin{array}{l}\text { Higher education } \\
\text { Health insurance }(n)\end{array}$ & 523 & 72 & 13.8 & 53 & 10.1 & 19 & 3.7 & \\
\hline Yes & 1648 & 297 & 18.0 & 168 & 10.2 & 129 & 7.8 & 0.001 \\
\hline No & 749 & 95 & 12.7 & 59 & 7.9 & 36 & 4.8 & \\
\hline \multicolumn{9}{|l|}{ Region $(n)$} \\
\hline Central & 1145 & 136 & 11.9 & 72 & 6.3 & 64 & 5.6 & \\
\hline Northern & 999 & 187 & 18.7 & 121 & 12.1 & 65 & 6.6 & 0.0001 \\
\hline Southern & 263 & 70 & 26.7 & 35 & 13.3 & 35 & 13.4 & \\
\hline \multicolumn{9}{|l|}{ Employment $(n)$} \\
\hline Yes & 277 & 46 & 16.8 & 25 & 9.2 & 21 & 7.6 & 0.883 \\
\hline No & 2122 & 345 & 16.3 & 202 & 9.5 & 143 & 6.8 & \\
\hline
\end{tabular}

aOn account of missing values not all categories of explanatory variables total 393. JD, Jordanian Dinars. 
Table 3 Logistic regression analysis on unmet need

\begin{tabular}{|c|c|c|c|}
\hline Explanatory variable & OR & $95 \% \mathrm{Cl}$ & $p$ \\
\hline $\begin{array}{l}\text { Respondent age } \\
\text { Living children (n) }\end{array}$ & & 0.01 \\
\hline$<3$ & 1.29 & $0.89-1.89$ & 0.18 \\
\hline $3-4$ & 0.86 & $0.63-1.17$ & 0.33 \\
\hline$\geq 5 \mathrm{a}$ & - & - & - \\
\hline \multicolumn{4}{|l|}{ Income quantiles (JD) } \\
\hline$\geq 130$ & 1.29 & $0.86-1.94$ & 0.22 \\
\hline $130-160$ & 1.18 & $0.79-1.76$ & 0.41 \\
\hline $160-200$ & 1.22 & $0.84-1.77$ & 0.30 \\
\hline $200-260$ & 0.71 & $0.44-1.15$ & 0.16 \\
\hline$>260^{a}$ & - & - & - \\
\hline \multicolumn{4}{|l|}{ Education } \\
\hline Illiterate & 2.06 & $1.22-3.48$ & 0.01 \\
\hline School & 1.25 & $0.90-1.74$ & 0.17 \\
\hline Higher educationa & - & - & - \\
\hline \multicolumn{4}{|l|}{ Health insurance } \\
\hline Yes & 1.29 & $0.97-1.71$ & 0.09 \\
\hline Noa & - & - & - \\
\hline \multicolumn{4}{|l|}{ Region } \\
\hline Central & 0.45 & $0.32-0.64$ & 0.00 \\
\hline Northern & 0.64 & $0.46-0.89$ & 0.01 \\
\hline Southerna & - & - & - \\
\hline \multicolumn{4}{|l|}{ Employment } \\
\hline Yes & 1.10 & $0.72-1.69$ & 0.66 \\
\hline $\mathrm{No}^{\mathrm{a}}$ & - & - & - \\
\hline
\end{tabular}

aComparison group. JD, Jordanian Dinars; OR, odds ratio.

purpose of birth spacing or limiting births) and selected demographic characteristics showed that with increasing age the prevalence of unmet need for spacing decreases. The prevalence of unmet need for birth spacing among women below the age of 25 years was $16.9 \%$, while it was 9.7\% for women aged $25-35$ years and $3.8 \%$ for women aged over 35 years. The pattern of unmet need prevalence for women who intended to limit births showed a rising prevalence with increasing age (Table 2). Similar patterns were seen when the number of living children was analysed. Table 2 shows that the prevalence of standard unmet need for spacing births for women with five or more children was $4.9 \%$ compared to $15.1 \%$ for women with unmet need for limiting births. No particular pattern was seen when income categories were examined. Moreover, when the educational level of women was analysed, with an increase in the level of education the prevalence of unmet need showed an upward trend for women who intended to space births and a downward trend for women who intended to limit births. There were also minor variations noted when health insurance and employment variables were studied (Table 2.) Both types of unmet need (i.e. birth limiting and spacing) showed higher prevalence figures in the southern region $(13.3 \%$ and $13.4 \%$ for birth spacing and limiting, respectively) and northern region $(12.1 \%$ and $6.6 \%$, respectively) when compared to the central region $(6.3 \%$ and $5.6 \%$, respectively).

Table 3 shows the results of the multivariate analysis using a logistic regression equation where the dependent variable was the unmet need and the independent variables were selected demographic characteristics. Age was found to play a significant role in the prediction of unmet need where an increase of 1 year in a woman's age contributes $4 \%$ to the likelihood that a woman has unmet need for family planning $(p<0.01)$. The number of living children and income were not significant predictors of unmet need (Table 3). Compared to women with higher education, illiterate women had a greater than two-fold increase in the risk of having unmet need $(p<0.01)$, while women with some form of schooling had a statistically insignificant $25 \%$ higher chance of having unmet need compared to women with higher education. Women in the central region had a two-fold lower chance $(p<0.01)$ of having unmet need when compared to women in the southern region (Table 3). However, women in the northern region had a 1.5 -fold lower chance of having unmet need when compared to women in the southern region.

\section{Discussion and conclusions}

The literature states a figure of $14.2 \%$ for the unmet need for family planning in Jordan in 1998. The most recent data from the 2002 JPFHS showed that the overall percentage of women who do not want any more children was $43.9 \%$. The same survey showed that the overall standard unmet need for family planning was $11 \%$, comprising $5.6 \%$ and $5.5 \%$ for birth spacing and limiting births, respectively.

In the present study the overall standard unmet need prevalence was $16.3 \%$, this being higher than the prevalence reported in the 2002 JPFHS. It is worthwhile mentioning that the present study is facility-based and limited to $\mathrm{MOH}$ centres as opposed to the JPFHS, which is a community-based study. Moreover, women who receive care from the $\mathrm{MOH}$ may have different socioeconomic characteristics and different reproductive health profiles from the general female population.

The relatively high rates of unmet need identified by the present study demand that MOH staff increase their efforts and pay special attention to these groups of women who are known to come to health facilities either when they are sick or for their children's growth monitoring and vaccinations.

The prevalence of unmet need remains high despite a wide network of comprehensive health centres and primary health centres, in addition to numerous projects that target Jordanian women as the recipients of information, education and communication materials. The current scenario in which women are expressing a desire not to have any more children and that fact that these women are not using family planning methods would appear to pave the way for potential health care delivery in the form of counselling at the very least. Clearly there is potential demand for family planning service delivery. Whilst increasing efforts to reach the $16 \%$ of women with unmet need, special attention needs to be paid to age, region and education as the main predictors for unmet need. Consequently, family planning programmes should target illiterate women in order to inform them about birth control measures. Efforts to strengthen family planning programmes in the southern region are recommended. Particular attention should be made to tailor family planning messages to women aged over 35 years who are currently non-users of family planning methods.

For this facility-based study, women who did not use MOH services had no chance of being selected to participate in the study. It is therefore likely that women non-users of services will require a different intervention through community outreach, since they are likely to have a worse fertility profile than the women targeted in the present study.

Finally, it is worthwhile noting that the non-static nature of unmet need (involving the interaction of fertility desire and contraceptive use) means that high or low prevalence of unmet need is not indicative of success or failure in family planning programmes. Accordingly, when developing an unmet need strategy, programme planners and decision-makers should consider the unmet need concept in respect of its various composites and correlates.

Statements on funding and competing interests

Funding None identified.

Competing interests None identified. 


\section{References}

1 Jordan Population and Housing Principal Report. Amman, Jordan: Department of Statistics, 2001.

2 Findings in the Jordan Fertility and Family Health Survey 1983. Published in the Proceedings of the Symposium on Population, Fertility and Family Health in Jordan, Amman, Jordan, 1983.

3 Jordan Fertility and Family Health Survey. Report of Principal Findings. Amman, Jordan: Department of Statistics, 1998.

4 Zoubi A, Poedjastoeti S, Ayad M. Jordan Population and Family Health Survey (JPFHS). 1990 Demographic and Family Health
Survey. Columbia, MD: Ministry of Health, Department of Statistics, IRD/MACRO International, 1992.

5 Annual Report of Health Indicators in Jordan. Amman, Jordan: Jordan Ministry of Health, 2001.

6 Jordan Population and Family Health Survey. Report of Key Findings. Amman, Jordan: Department of Statistics, 2002.

7 Westoff CF, Bankole A. Unmet Need: 1990-1994 (DHS Comparative Studies No. 16). Calverton, MD: Macro International, June 1995; 55.

\section{JOURNAL REVIEW}

Oral contraceptive use and cancer. findings in a large cohort study, 1968-2004. Vessey M, Painter R. J Cancer 2006; 95: 385-389

Following recent reports from the International Agency for Research on Cancer (IARC), concerns have been raised regarding the possible increased risk of various cancers relating to usage of the oral contraceptive (OC) pill. This large cohort study, leading on from previous reports from the Oxford Family Planning Association (FPA), set out to truly answer this question. Particular attention was focused on breast, cervical, uterine body and ovarian cancers and the potential beneficial effects on the latter two.

The study recruited and annually followed up women who attended UK family planning clinics from 1968 to 1974 . The women were all married, white and aged 25-39 years. Most other confounding factors were well accounted for in the analysis. The study recruited 17032 women, which totalled 540000 woman-years. Annual follow-up was conducted until the age of 45 years with only a $0.4 \%$ annual loss rate to follow-up. The researchers analysed the effect of both the duration and interval since cessation of usage of the OC. The relative rate (RR) of non-gynaecological cancers was not affected by either of these factors and no correlation was shown. Of particular note is the nil effect seen on breast cancer and slightly protective effect some 20 years after cessation. The RR of cervical cancers was strongly influenced by duration of usage, with the RR varying from 2.9 after 4 years' usage to 6.1 after 8 years. A profound lingering effect of the $\mathrm{OC}$ on cervical cancer was also seen with a RR of 5.2 seen at 4 years after cessation and still 4.6 after 8 years. A strongly protective effect of the OC was shown for both uterine body and ovarian cancers. This was seen with uterine body cancer regardless of the length of time the OC was taken whereas such an effect on ovarian cancer was only evident after 4 years of therapy. The protective effect also persisted well beyond cessation of treatment, with RR of only 0.5 (uterine) and 0.6 (ovarian) seen 20 years after cessation.

This study looked at OC products containing $50 \mathrm{\mu g}$ estrogen, which is relatively high for today's market. Therefore some effects seen here may not be as marked today. Naturally this study only deals with a streamed, predominantly Social Class I, health-seeking population but the use of RR instead of incidence does, I think, go a long way towards counteracting these and many confounders. When data were pooled on the three gynaecological cancers the RR in non-users versus users of the OC were 0.57 and 0.37 . In essence this study clearly shows the harmful effects of OC usage on the RR of cervical cancer but that this is outweighed by the protection offered from uterine body and ovarian cancers.

Reviewed by Paul Mills, MBChB, MRCS(Ed) SHO3 in Obstetrics and Gynaecology, Royal Infirmary of Edinburgh, Edinburgh, UK
Specialist contraceptive counselling and provision after termination of pregnancy improves uptake of long-acting methods but does not prevent repeat abortion: a randomized trial. Schunmann C, Glasier A. Hum Reprod 2006; 21: 2296-2303

This is an interesting and well-conducted randomised controlled trial powered to show a difference in uptake of post-termination contraception. Unfortunately only $53 \%$ of all eligible women were randomised and follow-up data were only available for $60 \%$ (control) and $63 \%$ (intervention) of the study participants.

The intervention, comprising a detailed interview/contraceptive counselling prior to or immediately after termination of pregnancy (TOP) and supply of contraception prior to discharge after TOP, led to increased uptake of contraception in the intervention group (271/316) compared to standard care $(115 / 297, p<0.001)$. This was particularly the case for uptake of longacting contraception (141 in intervention vs 78 in control group, $p<0.001)$. However, at 4 -month follow-up there was no longer a difference in overall use of or continuation of contraception nor was there any difference between the groups undergoing repeat abortion in the same hospital within the 2-year study period $(14.6 \%$ vs $10 \%$, $p=0.267$ ).

Changing contraceptive behaviour seems to need more than a single intervention and easy access to first supply of contraception even if using long-acting methods.

Reviewed by Eva Jungmann, MRCP, MSc Consultant Physician in Genitourinary Medicine/HIV, Archway Sexual Health Clinic, The Whittington Hospital, London, UK

Effects of raloxifene on cardiovascular events and breast cancer in postmenopausal women. Barrett-Connor E, Mosca L, Collins P, Geiger MJ, Grady D, Kornitzer M, et al.; Raloxifene Use for The Heart (RUTH) Trial Investigators. N Engl J Med 2006; 355: 125-137

The Raloxifene Use for the Heart (RUTH) trial was initiated in 1998. This was at a time when observational studies suggested a reduced incidence of coronary heart disease (CHD) in postmenopausal women receiving estrogen therapy. This was further supported by the favourable effects of selective estrogen-receptor modulators (SERMs) on serum lipid profiles. Initially the trial was designed to assess the effect of $60 \mathrm{mg}$ raloxifene on coronary events in women with already existing CHD or multiple risk factors.

A total of 10101 postmenopausal women (mean age, 67.5 years) participated in this international, multicentre, randomised, doubleblind, placebo-controlled trial. The median follow-up was 5.6 years. There were two primary outcomes in comparison to placebo: first, the incidence of coronary events (death from coronary cause, non-fatal myocardial infarction or hospitalisation for acute coronary syndrome) and second, the occurrence of invasive breast cancer.

Raloxifene is a non-steroidal SERM with estrogen-agonistic properties in the bone and estrogen-antagonistic properties in the endometrium and breast. Previous evidence from the MORE trial in postmenopausal women with osteoporosis demonstrated a reduction in the risk of invasive breast cancer and no increase in endometrial pathology. ${ }^{1}$ Additionally, raloxifene increased bone density in the spine and femoral neck with a reduction of vertebral but not hip fractures. ${ }^{2}$ This had to be balanced against the increased risk of VTE.

The primary outcomes of the RUTH trial were as follows. Women receiving raloxifene had no increase in death from coronary causes, nonfatal myocardial infarction or hospitalisation for acute coronary syndrome in comparison to women receiving placebo. Raloxifene did reduce significantly the incidence of invasive breast cancer - primarily estrogen-receptor-positive invasive breast cancer - by $55 \%$. Additionally, there was a significant risk reduction of clinical vertebral fractures by $35 \%$ but no reduction in non-vertebral fractures. These benefits have to be reviewed in the light of an increased risk of VTE $(44 \%)$ and fatal stroke (49\%). Other adverse events more commonly observed in the raloxifene group included hot flushes, peripheral oedema, gallbladder disease and leg cramps.

In summary, the RUTH trial confirms the benefits of SERMs in the reduction of invasive breast cancer and vertebral fracture. Raloxifene, in comparison to tamoxifen, does not increase endometrial pathology (confirmed in the MORE trial). Unfortunately these benefits have to be balanced against the increased risk of VTE and fatal stroke. Contrary to the initial trial design, a reduction in coronary events was not observed and therefore a cardio-protective effect cannot be assumed. Finally, the RUTH trial in comparison to the Women' Health Initiative trial did not include a 'global index'; the risks and benefits of SERM therapy should therefore be tailored to the ndividual needs of postmenopausal women.

References Nickelsen T, Genant HK, et al. Reduction of vertebral risk in postmenopausal women with osteoporosis treated with raloxifene: results from a 3 other . Raxis

2 Cummings SR, Eckert S, Krueger KA, Grady D, Powles TJ, Cauley JA, et al. The effect of raloxifene on risk of breast cancer in postmenopausal women: ( 281: 2189-2197; erratum in JAMA 1999; 282: 2124

Reviewed by Anja Guttinger, MRCOG, DFFP Subspecialty Trainee, Sexual and Reproductive Health, Family Planning and Well Woman Services, Dean Terrace Clinic, Edinburgh, UK 


\section{References}

1 Jordan Population and Housing Principal Report. Amman, Jordan: Department of Statistics, 2001.

2 Findings in the Jordan Fertility and Family Health Survey 1983. Published in the Proceedings of the Symposium on Population, Fertility and Family Health in Jordan, Amman, Jordan, 1983.

3 Jordan Fertility and Family Health Survey. Report of Principal Findings. Amman, Jordan: Department of Statistics, 1998.

4 Zoubi A, Poedjastoeti S, Ayad M. Jordan Population and Family Health Survey (JPFHS). 1990 Demographic and Family Health
Survey. Columbia, MD: Ministry of Health, Department of Statistics, IRD/MACRO International, 1992.

5 Annual Report of Health Indicators in Jordan. Amman, Jordan: Jordan Ministry of Health, 2001.

6 Jordan Population and Family Health Survey. Report of Key Findings. Amman, Jordan: Department of Statistics, 2002.

7 Westoff CF, Bankole A. Unmet Need: 1990-1994 (DHS Comparative Studies No. 16). Calverton, MD: Macro International, June 1995; 55.

\section{JOURNAL REVIEW}

Oral contraceptive use and cancer. findings in a large cohort study, 1968-2004. Vessey M, Painter R. J Cancer 2006; 95: 385-389

Following recent reports from the International Agency for Research on Cancer (IARC), concerns have been raised regarding the possible increased risk of various cancers relating to usage of the oral contraceptive (OC) pill. This large cohort study, leading on from previous reports from the Oxford Family Planning Association (FPA), set out to truly answer this question. Particular attention was focused on breast, cervical, uterine body and ovarian cancers and the potential beneficial effects on the latter two.

The study recruited and annually followed up women who attended UK family planning clinics from 1968 to 1974 . The women were all married, white and aged 25-39 years. Most other confounding factors were well accounted for in the analysis. The study recruited 17032 women, which totalled 540000 woman-years. Annual follow-up was conducted until the age of 45 years with only a $0.4 \%$ annual loss rate to follow-up. The researchers analysed the effect of both the duration and interval since cessation of usage of the OC. The relative rate (RR) of non-gynaecological cancers was not affected by either of these factors and no correlation was shown. Of particular note is the nil effect seen on breast cancer and slightly protective effect some 20 years after cessation. The $R R$ of cervical cancers was strongly influenced by duration of usage, with the RR varying from 2.9 after 4 years' usage to 6.1 after 8 years. A profound lingering effect of the $\mathrm{OC}$ on cervical cancer was also seen with a RR of 5.2 seen at 4 years after cessation and still 4.6 after 8 years. A strongly protective effect of the OC was shown for both uterine body and ovarian cancers. This was seen with uterine body cancer regardless of the length of time the OC was taken whereas such an effect on ovarian cancer was only evident after 4 years of therapy. The protective effect also persisted well beyond cessation of treatment, with RR of only 0.5 (uterine) and 0.6 (ovarian) seen 20 years after cessation.

This study looked at OC products containing $50 \mathrm{\mu g}$ estrogen, which is relatively high for today's market. Therefore some effects seen here may not be as marked today. Naturally this study only deals with a streamed, predominantly Social Class I, health-seeking population but the use of RR instead of incidence does, I think, go a long way towards counteracting these and many confounders. When data were pooled on the three gynaecological cancers the RR in non-users versus users of the OC were 0.57 and 0.37 . In essence this study clearly shows the harmful effects of OC usage on the RR of cervical cancer but that this is outweighed by the protection offered from uterine body and ovarian cancers.

Reviewed by Paul Mills, MBChB, MRCS(Ed) SHO3 in Obstetrics and Gynaecology, Royal Infirmary of Edinburgh, Edinburgh, UK
Specialist contraceptive counselling and provision after termination of pregnancy improves uptake of long-acting methods but does not prevent repeat abortion: a randomized trial. Schunmann C, Glasier A. Hum Reprod 2006; 21: 2296-2303

This is an interesting and well-conducted randomised controlled trial powered to show a difference in uptake of post-termination contraception. Unfortunately only $53 \%$ of all eligible women were randomised and follow-up data were only available for $60 \%$ (control) and $63 \%$ (intervention) of the study participants.

The intervention, comprising a detailed interview/contraceptive counselling prior to or immediately after termination of pregnancy (TOP) and supply of contraception prior to discharge after TOP, led to increased uptake of contraception in the intervention group (271/316) compared to standard care $(115 / 297, p<0.001)$. This was particularly the case for uptake of longacting contraception (141 in intervention vs 78 in control group, $p<0.001)$. However, at 4 -month follow-up there was no longer a difference in overall use of or continuation of contraception nor was there any difference between the groups undergoing repeat abortion in the same hospital within the 2-year study period $(14.6 \%$ vs $10 \%$, $p=0.267$ ).

Changing contraceptive behaviour seems to need more than a single intervention and easy access to first supply of contraception even if using long-acting methods.

Reviewed by Eva Jungmann, MRCP, MSc Consultant Physician in Genitourinary Medicine/HIV, Archway Sexual Health Clinic, The Whittington Hospital, London, UK

Effects of raloxifene on cardiovascular events and breast cancer in postmenopausal women. Barrett-Connor E, Mosca L, Collins P, Geiger MJ, Grady D, Kornitzer M, et al.; Raloxifene Use for The Heart (RUTH) Trial Investigators. N Engl J Med 2006; 355: 125-137

The Raloxifene Use for the Heart (RUTH) trial was initiated in 1998. This was at a time when observational studies suggested a reduced incidence of coronary heart disease (CHD) in postmenopausal women receiving estrogen therapy. This was further supported by the favourable effects of selective estrogen-receptor modulators (SERMs) on serum lipid profiles. Initially the trial was designed to assess the effect of $60 \mathrm{mg}$ raloxifene on coronary events in women with already existing CHD or multiple risk factors.

A total of 10101 postmenopausal women (mean age, 67.5 years) participated in this international, multicentre, randomised, doubleblind, placebo-controlled trial. The median follow-up was 5.6 years. There were two primary outcomes in comparison to placebo: first, the incidence of coronary events (death from coronary cause, non-fatal myocardial infarction or hospitalisation for acute coronary syndrome) and second, the occurrence of invasive breast cancer.

Raloxifene is a non-steroidal SERM with estrogen-agonistic properties in the bone and estrogen-antagonistic properties in the endometrium and breast. Previous evidence from the MORE trial in postmenopausal women with osteoporosis demonstrated a reduction in the risk of invasive breast cancer and no increase in endometrial pathology. ${ }^{1}$ Additionally, raloxifene increased bone density in the spine and femoral neck with a reduction of vertebral but not hip fractures. ${ }^{2}$ This had to be balanced against the increased risk of VTE.

The primary outcomes of the RUTH trial were as follows. Women receiving raloxifene had no increase in death from coronary causes, nonfatal myocardial infarction or hospitalisation for acute coronary syndrome in comparison to women receiving placebo. Raloxifene did reduce significantly the incidence of invasive breast cancer - primarily estrogen-receptor-positive invasive breast cancer - by $55 \%$. Additionally, there was a significant risk reduction of clinical vertebral fractures by $35 \%$ but no reduction in non-vertebral fractures. These benefits have to be reviewed in the light of an increased risk of VTE $(44 \%)$ and fatal stroke (49\%). Other adverse events more commonly observed in the raloxifene group included hot flushes, peripheral oedema, gallbladder disease and leg cramps.

In summary, the RUTH trial confirms the benefits of SERMs in the reduction of invasive breast cancer and vertebral fracture. Raloxifene, in comparison to tamoxifen, does not increase endometrial pathology (confirmed in the MORE trial). Unfortunately these benefits have to be balanced against the increased risk of VTE and fatal stroke. Contrary to the initial trial design, a reduction in coronary events was not observed and therefore a cardio-protective effect cannot be assumed. Finally, the RUTH trial in comparison to the Women' Health Initiative trial did not include a 'global index'; the risks and benefits of SERM therapy should therefore be tailored to the ndividual needs of postmenopausal women.

References Nickelsen T, Genant HK, et al. Reduction of vertebral retse oster oster . Raxis

2 Cummings SR, Eckert S, Krueger KA, Grady D, Powles TJ, Cauley JA, et al. The effect of raloxifene on risk of breast cancer in postmenopausal women: The randomized trial. 281: 2189-2197; erratum in JAMA 1999; 282: 2124.

Reviewed by Anja Guttinger

Subspecialty Trainee, Sexual and Reproductive

Health, Family Planning and Well Woman

Services, Dean Terrace Clinic, Edinburgh, UK 